\title{
El Aporte de las Nuevas Guías de la Sociedad de Vía Aérea Difícil (DAS)
}

\author{
RICARDO URTUBIA V. ${ }^{1}$, ELIANA ESCUDERO Z. ${ }^{2}$, ORLANDO ESPARZA G. ${ }^{3}$, \\ JOSÉ MIGUEL GUTIÉRREZ G. ${ }^{4}$
}

1 Anestesiólogo, Clínica Vespucio, Profesor Facultad de Medicina, Universidad Finis Terrae, FIDIVA Chile.

2 Enfermera, Matrona, Magister en Educación, Directora de la Escuela de Enfermería, Universidad Finis Terrae, Presidenta de la Sociedad Chilena de Simulación Clínica y Seguridad del Paciente (SOCHISIM).

3 Anestesiólogo, Complejo Asistencial Barros Luco y Clínica Vespucio, Docente de Postgrado Universidad Diego Portales.

4 Médico, Cirujano, Instructor en Simulación, Universidad Finis Terrae.

El abordaje de la vía aérea requiere de habilidades técnicas, pero también de las llamadas habilidades no-técnicas que permitan reconocer el contexto del paciente que requiere dicha intervención y sortear los problemas que nos presenta. Dado que tanto el paciente electivo como en el que requiere una cirugía de urgencia pueden presentar dificultades con el manejo de la vía aérea, su resolución requiere no sólo de la pericia del operador, sino que además de la colaboración efectiva de todo el equipo humano involucrado.

Con ocasión del encuentro mundial sobre manejo de la vía aérea (el World Airway Management Meeting - WAMM), que las agrupaciones más influyentes del mundo -la Society for Airway Management (SAM) de Estados Unidos y la Difficult Airway Society (DAS) del Reino Unido- organizaron en la ciudad de Dublín, Irlanda, en noviembre pasado, la DAS publicó sus nuevas guías para el "manejo de la intubación difícil no-anticipada en adultos".

Al igual que las guías de $2004^{2}$, estas nuevas guías de la DAS están enfocadas en la "intubación difícil no-anticipada" y no en la "vía aérea difícil", que ha sido el abordaje tradicional. Explícitamente enfatizan su enfoque en el manejo y no en la anticipación, que también ha sido la estrategia clásica para abordar el tema. Uno de los aspectos más notables de estas guías es que, sorprendente y provocativamente, hacen una fuerte referencia al Manejo de Recursos en Crisis (MRC - del inglés, Crisis Resource Management) al decir "Deténgase y Piense".

Desde el punto de vista técnico, estas guías proporcionan una serie de planes secuenciales a usar cuando falla la intubación traqueal, los cuales están diseñados para priorizar la oxigenación y reducir la posibilidad de trauma sobre la vía aérea con los intentos repetidos de inserción de dispositivos. Adicionalmente, enfatizan sobre la importancia de verbalizar los planes, las alternativas a considerar, las secuencias ya utilizadas, y sobre todo declarar explícitamente el fracaso en cada una de las estrategias para pasar a la siguiente.

Este artículo pretende dar una visión comentada de estas nuevas guías provenientes de posiblemente el grupo más avanzado en el tema del manejo de la vía aérea en el mundo. 


\section{¿Por qué el foco en la intubación difícil no- anticipada?}

Las guías de la $\mathrm{ASA}^{3}$, actualizadas en $2013^{4}$, abordan la vía aérea difícil anticipada y no-anticipada. Sin embargo, desde la publicación de las primeras guías en 2004, la DAS se ha centrado en la intubación difícil no-anticipada argumentando que los problemas con la intubación han sido la causa más frecuente de muertes relacionadas con Anestesia, fundamentalmente ligados al trauma tisular por el procedimiento.

En un estudio danés ${ }^{5}$ recientemente publicado con un universo de 188.044 pacientes sometidos a intervenciones electivas, hubo 3.391 intubaciones difíciles $(1,8 \%)$, de las cuales el 93\% no fueron anticipadas. Por otro lado, se predijo dificultad en 929 pacientes, de las cuales se anticipó efectivamente sólo el $25 \%$. Esto nos demuestra una vez más que no existe ningún sistema de anticipación confiable y que el problema de la intubación fallida sigue siendo lo relevante. Una razón agregada a la pobre predictibilidad de la evaluación preoperatoria es la reportada por el estudio NAP4 ${ }^{11}$, que es que la deficiente evaluación de la vía aérea contribuyó a los malos resultados. Las causas principales fueron: omisión, evaluación incompleta o fracaso en modificar el manejo en respuesta a los hallazgos de la evaluación.

Adicionalmente, siendo la intubación traqueal el "estándar", su fracaso pone al manejo en condición de alerta. A pesar de que cada vez con mayor frecuencia los dispositivos supraglóticos (DSG) han remplazado a la intubación endotraqueal en cirugías electivas, la implementación de estas técnicas no es sinónimo de control total de la vía aérea. Por lo tanto, la decisión de la DAS de enfocarse en la intubación difícil no-anticipada permite apuntar al centro del blanco.

\section{¿Una guía clínica más?}

Desde 1993 se publican periódicamente "guías", o mejor dicho "propuestas" de un grupo de expertos, que muchas veces fallan en su aplicación real por la resistencia que encuentran en las personas que debieran aplicarlas y también por la no disponibilidad de los dispositivos o equipos que en ellas se describen. En diversos estudios se señala entre un 60 a 70\% de adhesión. Burgers y col. ${ }^{6}$, han descrito las limitaciones que tienen las guías clínicas y los resultados de los estudios para traspasar sus recomendaciones y hallazgos a la práctica clínica. Las principales barreras que las guías tienen para su aplicabilidad son que requieren del aprendizaje de nuevas habilidades y que forman parte de un esquema complejo, por lo que su comprensión y retención se ven limitadas. Leentjens y Burgers ${ }^{7}$ concluyen que para que una guía tenga éxito debe en primer lugar estar dirigida a un grupo homogéneo de personas, en términos que compartan visiones comunes y se vean motivados a adoptarlas no sólo como una imposición, sino como parte de una estrategia de seguridad que tenga sentido para ellos.

El estudio NAP4 ${ }^{11}$ mostró también que nos falta traspasar los resultados de los estudios a la implementación de conductas que mejoren la seguridad de los pacientes. Tal como lo demuestran diversos análisis, la adherencia real a las recomendaciones de las guías clínicas es baja.

El rechazo a las nuevas tendencias y permanecer refugiado en lo aprendido y considerado como paradigma (socialmente aceptado) es el llamado "efecto Semmelweis". Muchas veces, las nuevas recomendaciones implican un cambio cultural en el quehacer del equipo. Para que este cambio se produzca sin un gran rechazo requiere perseverancia y humildad, trabajo de educación y liderazgo.

Tomando en consideración estas reflexiones y también la evidencia sobre la eficacia y seguridad de los distintos procedimientos, estas nuevas guías han tratado de ponerse del lado del mundo real para formular sus recomendaciones. No describen técnicas muy complejas, sólo las que pueden llevar a resolver la crisis de la intubación fallida y con el menor riesgo posible.

\section{Factores Humanos}

La Organización Mundial de la Salud, publicó sobre el "factor humano" el año 2008, describiéndolo como el campo que concierne la interacción del ser humano con el sistema en el cual trabaja. Esto implica la administración de tareas, el trabajo en equipo, la percepción de riesgo, y la toma de decisiones entre otras, todas habilidades 
entrenables y especialmente en escenarios simulados. Señala también esta publicación que el entrenamiento de habilidades no técnicas es vital para reducir el error en la industria en general. No obstante, esto no ha sido aún incorporado en el trabajo en salud ${ }^{8}$.

En 2005 se produjo en Australia el triste caso de la Sra. Elaine Bromiley, quien falleció como consecuencia de un mal manejo de la situación "no puedo intubar - no puedo oxigenar"*. Históricamente, sólo desde 1993 nos hemos preocupado colectiva y estructuradamente de este tema a través de la publicación y difusión de guías, y de la organización de cursos de capacitación. Sin embargo, este caso abrió la mente de dos australianos, Nicholas Crimes (anestesiólogo) y Peter Fritz (urgenciólogo) y los llevaron a formular un nuevo enfoque para el manejo de las crisis relacionadas con la vía aérea. Así nació el "Vortex Approach", que contiene la muy agradable "zona verde", que permite pensar, reflexionar, considerar opciones, pedir ayuda, etc.

La instrucción de "deténgase y piense" también ofrece la oportunidad de comprender el problema que nos presenta esa vía aérea. Estos "segundos" de reflexión y discusión nos pueden evitar "minutos" de inacción o de nuevos errores. El esposo de Elaine, Martin Bromiley, piloto de aviación comercial con gran experiencia en el enfrentamiento de crisis, rápidamente relacionó las causas del desastre producido con su esposa con los factores presentes en los desastres aéreos, en los que el factor humano está presente en un $75 \%$ de los casos. Él dice que los problemas que llevaron al fatal desenlace fueron principalmente la falta de comunicación efectiva, la falta de liderazgo y una falla en la toma de decisiones. En los interrogatorios, los involucrados declaraban que no podían comprender por qué a pesar de que sabían perfectamente lo que tenían que hacer, que tenían el entrenamiento y el equipamiento necesarios, finalmente no lo hicieron.

En un estudio sobre el factor humano, Flin y col. ${ }^{10}$, identificaron elementos latentes en el equipo tratante, tales como mala comunicación, falta de entrenamiento, trabajo en equipo deficiente, y sistemas y procesos inadecuados, entre otros, que predisponen a perder la conciencia de lo que debía hacerse y que puede llevar a una mala toma de decisiones.

En lo concerniente a la comunicación, estas guías enfatizan que la "vía aérea difícil" debe ser declarada explícitamente para que así sea reconocida por todos los integrantes del equipo. A menudo se reconoce que el ser humano "ve lo que quiere ver", que "mira sin ver" y que "oye sin escuchar". Por lo tanto, una declaración explícita de "ies una vía aérea difícil!" aumenta la posibilidad de un cambio colectivo de mentalidad y una toma de conciencia sobre la situación.

En segundo lugar, estas guías han acogido los componentes del manejo de recursos en crisis que consideran fundamentalmente la comunicación efectiva y el trabajo en equipo. Esto implica que los integrantes del equipo tratante no sólo deben tener destrezas manuales, sino también destrezas verbales y de interprofesionalidad, cual muestra que el éxito o el fracaso dependen no sólo de los líderes sino que de la interacción de todo el equipo. Hoy, cada vez con mayor fuerza los equipos de Anestesia, Medicina Intensiva y Emergenciología están utilizando las herramientas del manejo de recursos en crisis, de manera que estas nuevas guías están en lo correcto al ponerse a tono con la evolución de los tiempos y competencias, lo cual en cierta modo cambia el paradigma de que lo que el líder ve es la única verdad y que ninguna otra opinión es aceptable.

En el caso de Elaine Bromiley, las enfermeras no se atrevieron a decir nada a los especialistas a pesar de que ellas trajeron el equipo de cricotirotomía y anunciaron su disponibilidad. ¿Por qué? Tal vez tenían miedo de decir algo incorrecto o muy osado, o a que los doctores les respondieran de manera agresiva. Quizás las razones sean muchas. Lo concreto es que este caso nos demuestra que a pesar de que trabajamos juntos, en las crisis no logramos la interprofesionalidad y el trabajo en equipo que se requiere.

El reporte del estudio NAP4 (National Audit Project $)^{11}$ publicado en 2011, sobre las complicaciones relacionadas con el manejo de la vía aérea tanto en Anestesia, en la UCI y en el SU,

* Nota: desde hace tiempo se reemplazó la expresión "no puedo intubar-no puedo ventilar" (NPI-NPV) por "no puedo intubar-no puedo oxigenar" (NPI-NPO). 
está representado en la forma en que estas guías han sido formuladas. Este estudio prospectivo de 1 año (2008-2009) nos ha demostrado que efectivamente, el manejo de la vía aérea está asociado a morbimortalidad. Sin embargo, más allá de las cifras sobre malos resultados, NAP4 ${ }^{11}$ nos mostró también que al menos el $40 \%$ de las causas subyacentes eran de origen no-técnico; es decir, el factor humano estaba implicado en buena parte de ellos.

Esta mirada psicológica también nos da una luz acerca de por qué muchas veces no pedimos ayuda o nos mostramos renuentes a ayudar si no se nos pide explícitamente ${ }^{12}$. Estas nuevas guías nos alientan a considerar estos factores humanos tanto a nivel individual, colectivo y organizacional para lograr el objetivo final que es mejorar la seguridad de los pacientes sometidos a las intervenciones sobre la vía aérea.

\section{Evaluación y Planificación preoperatorias}

La evaluación previa del paciente es adecuada sólo si se consideran los 4 abordajes de la vía aérea, intentando anticipar dificultades: la ventilación con máscara facial, el uso de dispositivos supraglóticos, la intubación traqueal y el abordaje cervical. De esta manera, se puntualiza que el anestesiólogo debe tener una estrategia definida antes de la inducción anestésica. Esta estrategia debe no sólo estar en la mente de este profesional, sino que debe ser discutida y compartida con el equipo colaborador en el período de "pausa de seguridad". El resultado de esta planificación debe derivar en la formulación de los planes A, $\mathrm{B}, \mathrm{C}$ y D descritos en las guías.

Adicionalmente, es importante utilizar una lista de chequeo de todos las variables involucradas en la inducción anestésica. La estrategia Vortex la considera como paso esencial antes de proceder:

- Preparación de las intervenciones: acceso intravenoso, drogas, monitorización.

- Prevención de la hipoxia: preoxigenación, oxigenación apneica, reoxigenación, posicionamiento, aspiración, y dispositivos para los cuatro abordajes de la vía aérea.

- Promoción del trabajo en equipo: supervisión, ayuda adicional, roles, planes.

Las guías enfatizan acerca de lo fundamental de la comunicación como pilar sobre el que se sostiene el desempeño individual y colectivo.

\section{Intubación en secuencia rápida (ISR)}

Sobre este tema, las guías se refieren a la preferencia entre succinilcolina y rocuronio, en cuanto a la rapidez del inicio de acción. Ya se ha establecido que la succinilcolina, además de sus efectos adversos conocidos, ofrece un tiempo seguro de apnea menor que el rocuronio (100 segundos de diferencia) debido a que la fasciculaciones que produce aumentan el consumo de oxígeno ${ }^{13,14}$. También que la dosis de rocuronio de $1,2 \mathrm{mg} \mathrm{kg}^{-1}$ ofrece un tiempo de acción similar a la succinilcolina, con el agregado de que su efecto es susceptible de ser antagonizado por sugammadex. Sin embargo, puntualizan que no es garantía poder recuperar la permeabilidad de la vía aérea y tampoco de la ventilación espontánea ${ }^{15}$.

También hacen referencia a la presión cricoidea (maniobra de Sellick), aunque su aplicación en ISR es todavía materia de debate. Algunos creen en su efectividad para evitar la aspiración de contenido gástrico, otros creen que debiera ser abandonada debido a que no hay evidencia acerca de su eficacia real, sus beneficios y posibles complicaciones ${ }^{16}$. Las guías concluyen con este respecto que si el operador encuentra dificultad durante la intubación o inserción de un DSG, debe liberar la presión cricoidea, manteniendo control laringoscópico y con un dispositivo de aspiración disponible. Adicionalmente, si se observara regurgitación, la maniobra debe ser reaplicada inmediatamente. Otras guías, tales como la alemana ${ }^{17}$ y la escandinávica ${ }^{18}$, dejan su uso o no al juicio clínico individual más que recomendar su uso de manera mandatoria. En EE.UU., la presión cricoidea ha sido removida de las guías 2010 de la American Heart Association ${ }^{19}$ (AHA) $y$ de la Eastern Association for the Surgery of Trauma $^{20}($ EAST) para la intubación traqueal de urgencia. Las muy completas recomendaciones de la SACH no mencionan este punto ${ }^{21}$.

Desde hace ya algún tiempo, en el mundo de la Medicina de Urgencia se viene hablando de un nuevo concepto con relación al manejo de la vía aérea en el paciente crítico: "la vía aérea fisiológicamente difícil" ${ }^{22}$. Este nuevo enfoque persigue evitar la morbimortalidad asociada a las 


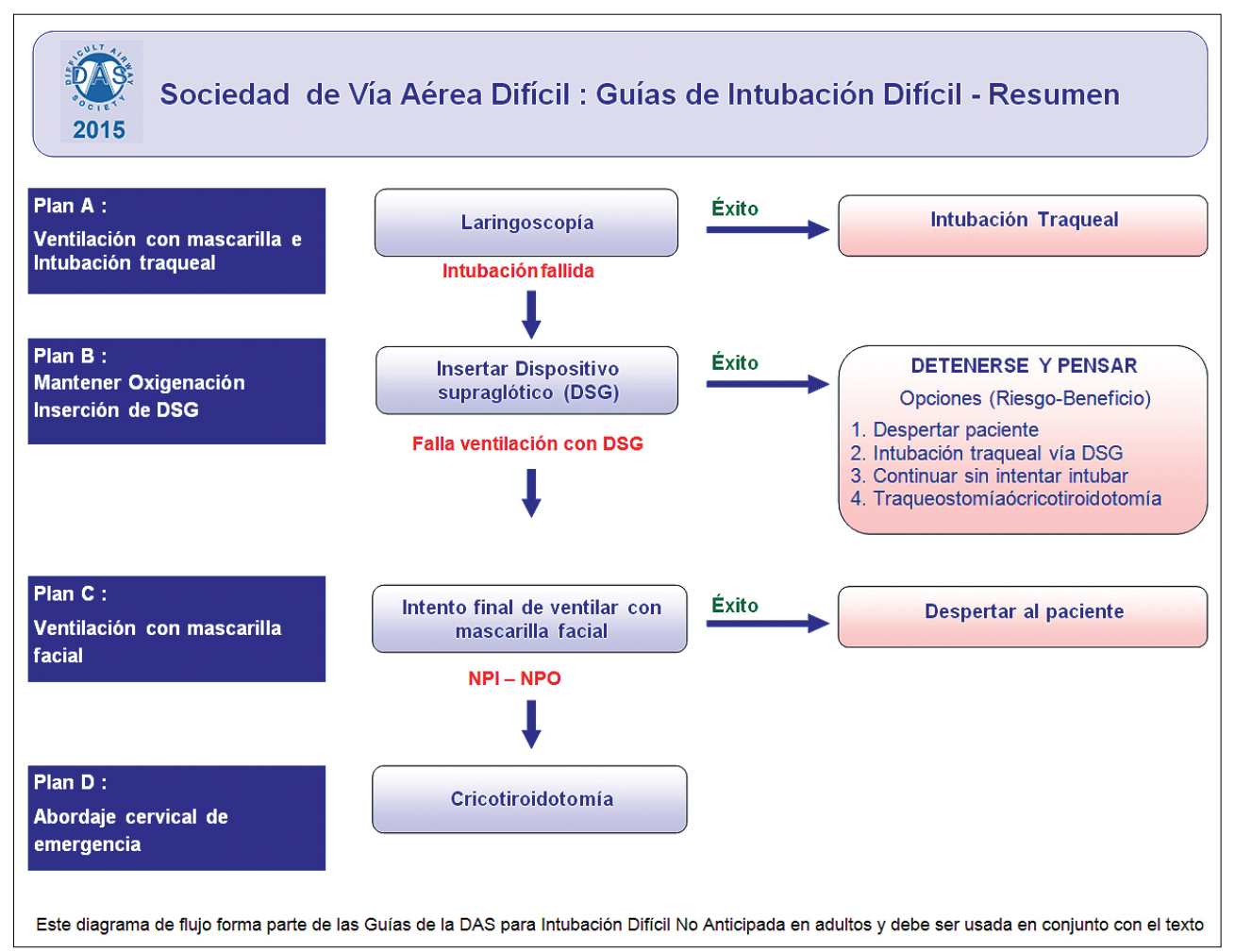

Figura 1.

alteraciones fisiológicas (hipoxemia, hipotensión, acidosis metabólica severa y falla ventricular derecha) del paciente enfrentado al efecto de drogas $\mathrm{y}$ de la ventilación a presión positiva ${ }^{23}$. Por esta razón, la estrategia de "intubación en secuencia rápida" está mutando a la "intubación en secuencia retardada" "24,25,26, que proporciona además una estrategia de "sedación para procedimiento" con el objeto de permitir la compensación hemodinámica y de oxigenación que necesita el paciente crítico antes de ser sometido a las intervenciones que requiere. En el mismo sentido, estas guías incluyen estos mismos conceptos, dando mucho énfasis a las nuevas estrategias de preoxigenación y oxigenación continua. Recalcan también la importancia de la planificación, optimización y de evitar los intentos repetidos de intervención, los cuales están relacionados con complicaciones importantes ${ }^{27}$. Estas nuevas estrategias constituyen hoy día un campo de investigación que está en desarrollo y posiblemente se convertirán en cotidiano en un futuro próximo. Es lo que las nuevas generaciones necesitan; romper con viejos paradigmas y navegar por la eficacia y la seguridad.

\section{Plan A: Máscara facial e Intubación traqueal}

La esencia del plan A es maximizar la probabilidad de la intubación exitosa "al primer intento" $\mathrm{y}$, en caso de fracaso, limitar el número y duración de los intentos de laringoscopía para evitar producir trauma que pueda llevar a la situación NPI-NPO. En este plan se puntualiza la importancia tanto de la posición del paciente como de la preoxigenación, así como del uso de relajantes neuromusculares para facilitar la ventilación con máscara facial.

Este punto nos puede resultar controversial, pues hemos aprendido que no es adecuado relajar al paciente sin comprobar que lo podremos ventilar con la máscara facial. Actualmente hay mucha evidencia de que el no uso de bloqueadores neuromusculares puede impedir la ventilación con máscara facial y que no debe darse por fracasada la técnica sin su uso ${ }^{28,29}$. Tampoco debe hacerse nuevos intentos de intubación sin relajación muscular.

En cuanto a la posición del paciente, la "posición de olfateo" descrita por Sir Ivan Magill 
Manejo de Intubación Traqueal Difícil No Anticipada en Adultos

\begin{tabular}{|c|}
\hline Plan A: Ventilar c/ mascarilla e intubar \\
\hline $\begin{array}{l}\text { Optimizar posición cabeza y cuello } \\
\text { Preoxigenar } \\
\text { Bloqueo neuromuscular adecuado } \\
\text { Laringoscopia (o video): Máximo } 3 \text { intentos } \\
\text { Manipulación laríngea externa } \\
\text { Bougie } \\
\text { Retirar presión cricoidea(Sellick) } \\
\text { Mantener oxigenación y anestesia }\end{array}$ \\
\hline
\end{tabular}

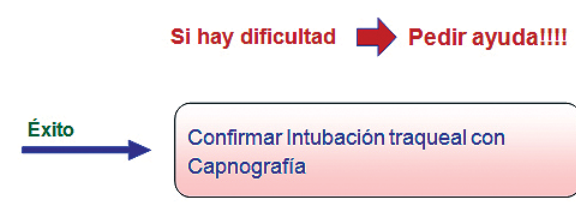

Plan B: Mantener Oxigenación-Insertar DSG

Preferir DSG de $2^{\mathrm{a}}$ generación

Cambiar dispositivo o tamaño: Máximo 3 intentos

Oxigenar y Ventilar

Declarar Intubación Fallida

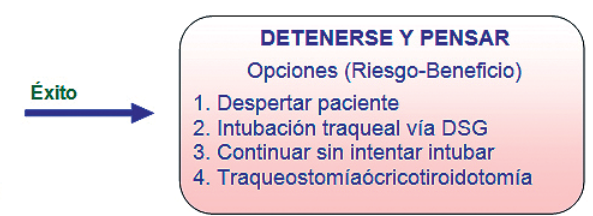

Plan C: Ventilación con máscara facial

Si es imposible ventilar : relajación muscular
profunda

Intento FINAL de ventilación con mascara facial

Ventilación con 2 operadores

Declarar NPI - NPO

\section{Plan D : Abordaje cervical de emergencia}

Cricotiroidotomía con bisturi

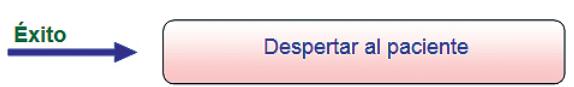

Cuidados y Seguimiento

Post-operatorio

- Monitorizar complicaciones

- Completar reporte vía aérea - Registrar

- Explicación verbal y escrita al paciente

- Reportar en base de datos

en 1936 sigue siendo la recomendada, habiendo sido comprobada su utilidad con estudios imagenológicos. Esta maniobra aumenta el espacio hipofaríngeo y favorece la ventilación con máscara facial. En pacientes obesos se recomienda la posición "en rampa", de manera que la horquilla esternal quede al mismo nivel que el conducto auditivo externo, situándose la cara en un nivel anterior a la línea horizontal que una ambos reparos anatómicos.

También se enfatiza la necesidad de preoxigenar a todos los pacientes antes de la inducción anestésica, con el objeto de retardar la desaturación de la hemoglobina y permitir disponer de más tiempo para maniobrar en caso de dificultad. Adicionalmente, la preoxigenación con la cabeza elevada en $20-25^{\circ}$ y el uso de dispositivos que proporcionen presión continua de oxígeno sobre la vía aérea, han demostrado un mayor tiempo antes de la desaturación en obesos.

Junto con lo anterior, estas guías por primera vez acogen la técnica de la oxigenación continua que, usando una naricera con flujo de oxígeno de 15 litros/min, fue postulada por Weingart y Levitan para pacientes críticos en el SU ${ }^{30}$. Esta técnica ha demostrado extender el período de apnea segura (tiempo en que la $\mathrm{SpO}_{2}$ demora en caer hasta $90 \%$ ). Asimismo, el uso de cánulas nasales con alto flujo de oxígeno humidificado (hasta 70 litros/min) ha demostrado extender el tiempo de apnea segura en obesos y en pacientes con vía aérea difícil ${ }^{31}$. Estas guías avalan la utilización de mascarillas de no-recirculación (con bolsa de reservorio) más naricera, ambas a 15 litros $/ \mathrm{min}$, en pacientes de alto riesgo. Sin embargo, surge la duda acerca del efecto dilucional que este flujo elevado de oxígeno produciría en el halogenado utilizado, lo cual puede producir un nivel inadecuado de profundidad anestésica. Una solución podría ser administrar un bolo adicional de propofol o utilizar TIVA durante ese período.

En cuanto a la elección del laringoscopio, el 
estándar es la laringoscopía directa tradicional, especialmente la que utiliza la hoja Macintosh (diseñada en 1942). Hasta el momento, no existe evidencia suficiente que indique que el videolaringoscopio (de los cuales hay varios modelos con diferentes características) deba remplazar a la laringoscopía directa en pacientes con vía aérea normal o difícili ${ }^{32}$, aunque nuevos estudios acumulan información en su favor ${ }^{33}$. Los videolaringoscopios ofrecen una mejor laringoscopía comparada con los laringoscopios tradicionales y también permiten una mejor interacción con los ayudantes, por lo que las guías recomiendan que en los servicios estén disponibles y que los anestesiólogos mantengan una práctica regular con ellos.

Por otro lado, también recomiendan tímidamente la manipulación externa de la laringe, que puede ser útil en la laringoscopía directa. Levi$\tan ^{34}$ ha acuñado el término "laringoscopía bimanual", queriendo decir que la laringoscopía es un proceso dual que comienza con la introducción del laringoscopio y elevación de la epiglotis para obtener algún grado de visualización de la glotis. El complemento y segunda fase es la manipulación externa de la laringe, descrita en 1993 por Knill $^{35}$ como maniobra BURP (Back-Up-RightPressure), aunque sin esta imbricación con el uso y rol del laringoscopio.

El videolaringoscopio ayuda en esta maniobra al permitir al ayudante que mantiene la posición del cartílago tiroides, ver los efectos de su accionar.

\section{Plan B: Mantener la oxigenación: inserción de un dispositivo supraglótico (DSG)}

El énfasis está en el uso de estos dispositivos para mantener la oxigenación y en la preferencia de los modelos de segunda generación pues ofrecen un sello mejorado, mayor seguridad frente a una eventual regurgitación de contenido gástrico y a su compatibilidad con las técnicas fibroscópicas de intubación.

El estudio NAP4 ${ }^{11}$ mostró que el $56 \%$ de las complicaciones se relacionaron con el uso de DSG y que la causa más frecuente fue su uso inadecuado. De hecho, ellos encontraron una tasa sorprendentemente alta de falla de la mascara laringea, 1:50. Aunque en el momento de realización del estudio el uso de DSG de segunda generación era incipiente, y por lo tanto, se desconoce su incidencia de complicaciones, las guías recomiendan su uso porque consideran que brindan mejor protección de la vía aérea. Aunque sólo i-gel, ML Proseal y ML Supreme tienen estudios importantes en adultos, en este momento se desconoce cuál es su rol y eficacia en los casos de vía aérea difícil.

Sin embargo, si bien mencionan algunos dispositivos y recomiendan los DSG de segunda generación, nos parece que al igual que otras guías de otros orígenes sólo mencionan DSG del tipo máscara laríngea (ML). Puntualmente, no consideran por ejemplo al Tubo Laríngeo (TL, VBM Medizintechnik GMBH, Sulz, Alemania), que también produce un excelente sello y brinda protección frente a la regurgitación de contenido gástrico ${ }^{36}$.

El mecanismo de funcionamiento del TL (al igual que el Combitubo) consiste en producir una cámara en la hipofaringe con sello proximal y distal, que permite la ventilación a través de una apertura situada entre ambos balones. Este mecanismo, diferente del de los DSG con sello perilaríngeo, condiciona también distintas causas de falla: los similares a ML fallan por presión excesiva de la vía aérea, mientras que el TL falla por problemas de posición durante la inserción.

El nuevo iLTS permite avanzar un tubo convencional a través de su lumen ${ }^{37}$, por lo que también es compatible con técnicas fibroscópicas de intubación. Adicionalmente, los modelos pediátricos son comparables a la $\mathrm{ML}^{38}$, y parecen tener un buen desempeño incluso en niños pequeños ${ }^{39}$. Por consiguiente, parece prudente considerar bajo el término "DSG" a la ML Supreme y al TL, como alternativas para la intubación-ventilación fallidas. Aunque publicadas en 2008, las guías españolas recomiendan el uso del Combitubo y del Easytube como alternativas no invasivas tras el eventual fracaso de la ML y/o Fastrach ${ }^{40}$.

Los dispositivos son diversos en su diseño y eficacia, por lo que es necesario considerar varios factores para determinar cuál utilizar en una situación dada, incluyendo las características anatómicas y fisiológicas del paciente, el escenario que se enfrenta, el nivel de habilidades del operador y la disponibilidad del equipamiento, entre otros. Cada dispositivo tiene características 
propias que pueden ser ventajosas en algunas situaciones y desventajosas en otras ${ }^{41}$.

\section{Plan C: Intento final de ventilación con más- cara facial}

Al igual que la versión de 2004, la recomendación va hacia atrás, volviendo a la máscara facial. Se considera que aunque la ventilación con máscara facial haya sido fácil, difícil o imposible, la situación pudiera haber cambiado tras los intentos frustros de intubación e inserción de DSG. De esta manera, si se logra ventilar se aconseja despertar al paciente, para lo cual se requerirá antagonizar el bloqueo neuromuscular. Si por el contrario, no se logra ventilar, se debe proceder inmediatamente con el Plan D, manteniendo los intentos por oxigenar al paciente.
Este enfoque parece razonable sólo si la ventilación con máscara facial ha sido efectiva en el Plan A, por lo que si hemos llegado a la situación NPI-NPO habiendo también fallado el videolaringoscopio y otro DSG alternativo, lo que parece más razonable es proceder inmediatamente con el Plan D.

\section{Plan D: Abordaje cervical de urgencia}

Este escenario se produce cuando todos los demás abordajes han fracasado. En la versión anterior, este Plan D retrocedía para utilizar nuevamente la ML como último intento.

El estudio NAP4 reportó algunos problemas con este abordaje desde el punto vista del $\mathrm{MRC}$, especialmente el retraso en la toma de la decisión, desconocimiento del equipo y de su

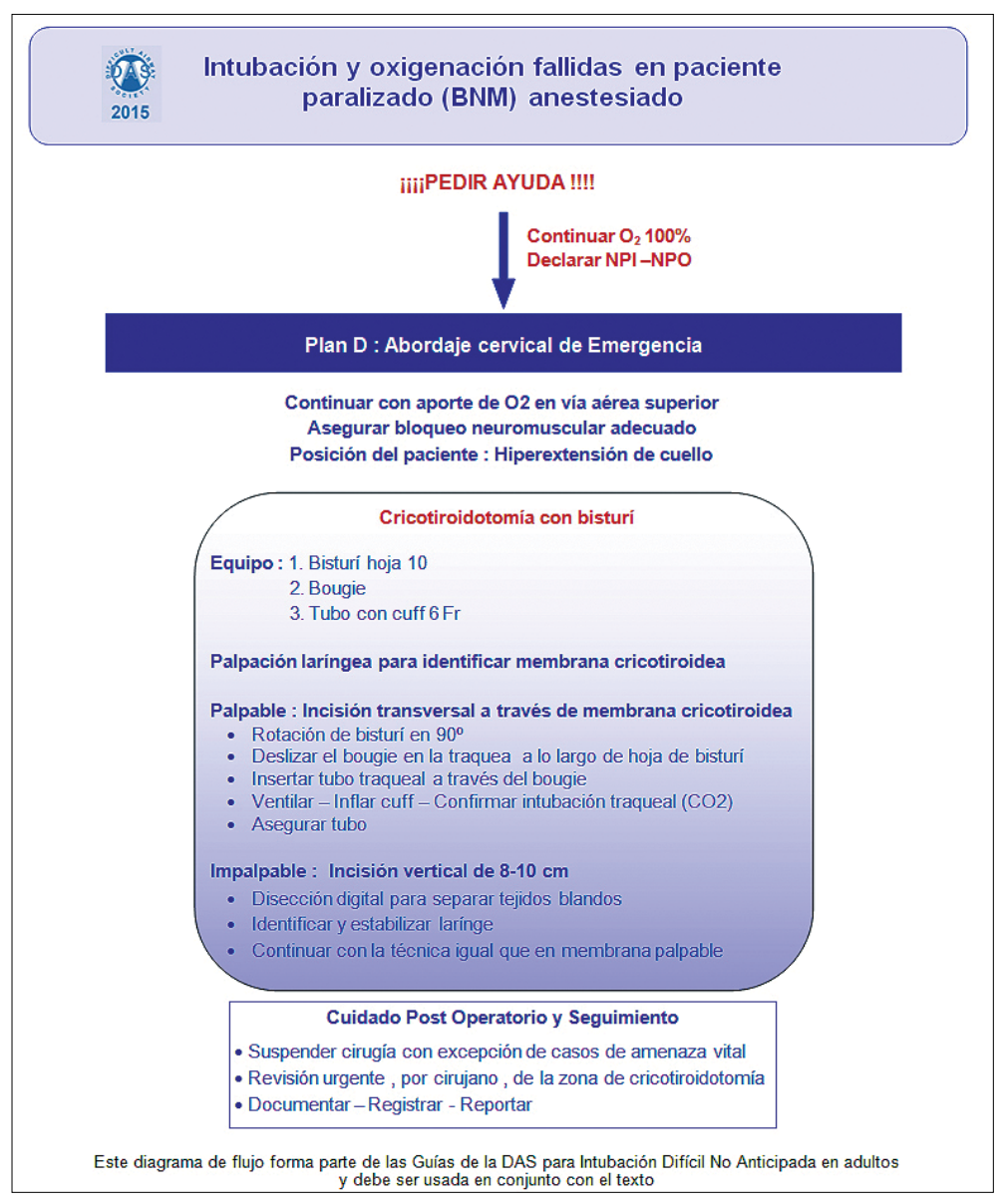

Figura 3. 
funcionamiento, no disponibilidad del equipo y problemas técnicos del procedimiento. Por esta razón, las guías pretenden acelerar la toma de decisión en una situación en que el tiempo es crucial. Nuevamente el estudio NAP4 revela que si se toma la cifra baja de $\mathrm{SpO}_{2}$ como gatillante, el tiempo que nos tome realizar el procedimiento será el mismo en el cual el cerebro estará severamente hipóxico, y que cuando se ha tomado la decisión, ya es muy tarde.

Las nuevas guías recomiendan una cricotirotomía mediante un incisión, aunque reconoce que la evidencia para recomendar esta técnica es limitada y que no excluye las técnicas por punción. Su consideración es utilizar un plan simple para rescatar la vía aérea, con equipamiento familiar y técnicas ensayadas para mejorar las probabilidades de éxito (tubo traqueal 6.0 DI, bisturí \#10 de hoja ancha y un bougie $)^{4243}$. El argumento está dado por el $63 \%$ de fracaso de la técnica por punción hecha por anestesiólogos encontrada en el estudio NAP4, y que frente a un situación que es muy infrecuente, se disponga inmediatamente del equipamiento necesario.

Sin embargo, NAP4 refiere que los anestesiólogos prefieren casi exclusivamente la técnica por punción y no reporta nada acerca del éxito o fracaso de la técnica quirúrgica hecha por anestesiólogos. La observación en talleres con tráqueas de cordero con más de 10.000 intentos, ha mostrado que en este modelo la técnica quirúrgica fallida dificulta mucho los posteriores intentos usando una cánula. El objetivo de la técnica con cánula, que es la aspiración de aire desde el lumen de la tráquea, puede no lograrse porque el lumen de la cánula se puede llenar de sangre, presente tras el intento quirúrgico previo. Por el contrario, un intento fallido con cánula no dificulta un posterior intento con bisturí.

En nuestro medio existen equipos que incluyen las dos técnicas (Melker, Surgicric III), y en lugares o situaciones sin grandes recursos es posible implementar la estrategia sugerida por las guías. Lo más importante es que los anestesiólogos, urgenciólogos e intensivistas nos entrenemos en esta técnica para hacerla eficaz y segura ${ }^{44}$.

\section{Manejo postoperatorio y seguimiento}

Estas guías también mencionan la necesidad de discutir lo ocurrido al interior del equipo, durante el período de "sign-out". Asimismo, como existe una correlación entre intubación difícil y trauma sobre la vía aérea, el paciente debe ser sometido a un período de observación para detectar complicaciones tardías. Cualquier instrumentación sobre la vía aérea puede producir daño, lo cual ha sido reportado con el uso de tubo traqueal, DSG, videolaringoscopios y fibroscopios. El sistema de reporte de complicaciones de la ASA indica que los sitios de lesión más frecuentes con la faringe y el esófago, las cuales son de difícil diagnóstico.

Finalmente, recomiendan que cualquier intubación fallida, acceso cervical de urgencia o ingreso a UCI por problemas relacionados con la vía aérea, deben ser notificadas y discutidas en comités ad hoc.

\section{Conclusiones}

¿Qué plantean de nuevo estas guías? En primer lugar, la planificación, que debe ser clara y definida al interior del equipo. Esta estrategia se enmarca bien de acuerdo a la idea de "equipo efectivo" propuesta por el estudio NAP5 ${ }^{45}$.

En segundo lugar está la "intubación al primer intento", lo cual implica que el primer intento (de un máximo de 4, el último por el más experto disponible) debe ser realizado en las mejores condiciones posibles, por lo que se debe optimizar la posición de todos los pacientes.

En tercer lugar, la importancia de la preoxigenación y de mantener la oxigenación durante el período de apnea, que necesariamente se produce durante la laringoscopía-intubación.

En cuarto lugar, la necesidad de disponer de videolaringoscopios y de mantener un cierto grado de entrenamiento en su uso.

En quinto lugar, declarar el fracaso con celeridad y claridad, de modo que puedan implementarse rápidamente las estrategias para rescatar esa vía aérea. Las opciones son cuatro: 1) despertar al paciente; 2) intentar una intubación fibroscópica (no a ciegas) a través de un DSG; 3 ) proceder con la cirugía ventilando con un DSG, y 4) proceder con la vía aérea quirúrgica ventilando con una máscara facial y adyuvantes.

Una limitación de estas nuevas guías es que no son muy explícitas en formular un arsenal de 
dispositivos de acuerdo a estrategias publicadas por la misma $\mathrm{DAS}^{46}$.

Estas nuevas guías de la DAS no sólo proporcionan un consenso valioso acerca de los aspectos técnicos, con nuevos enfoques centrados en la seguridad del paciente. No es un algoritmo más, sino una estructuración basada en las personas, en la realidad del medio. En este sentido, enfatizan que nosotros debemos hacernos cargo del impacto profesional, ambiental y sicológico de nuestro actuar ${ }^{47}$.

Estas guías no sólo nos han invitado a "detenernos y pensar" para resolver un problema agudo y apremiante, sino que también nos instan a que debemos "detenernos y pensar" en cómo plasmar todos estos elementos en un cambio en nosotros que sea significativo, para tratar de brindar la mayor seguridad posible a nuestros pacientes.

Finalmente, quisiéramos citar nuevamente a Martin Bromiley, quien escribe la siguiente frase en el prólogo del estudio NAP4 ${ }^{11}$ : "Dennos las herramientas para que hacer lo correcto sea fácil, dennos lo procesos para que la seguridad tenga la mejor chance, y dennos el entrenamiento para que los podamos usar y podamos conducirnos para dar un salto cuántico hacia una práctica más segura".

\section{Referencias}

1. Frerk C, Mitchell VS, McNarry AF, Mendonca C, Bhagrath R, Patel A, et al. Difficult Airway Society intubation guidelines working group. Difficult Airway Society 2015 guidelines for management of unanticipated difficult intubation in adults. $\mathrm{Br}$ J Anaesth 2015 Dec;115(6):82748.

2. Henderson JJ, Popat MT, Latto IP, Pearce AC; Difficult Airway Society. Difficult Airway Society guidelines for management of the unanticipated difficult intubation. Anaesthesia 2004 Jul;59(7):675-94.

3 . Practice guidelines for management of the difficult airway. A report by the American Society of Anesthesiologists Task Force on Management of the Difficult Airway. Anesthesiology 1993 Mar;78(3):597-602.

4. Apfelbaum JL, Hagberg CA, Caplan RA, Blitt CD, Connis RT, Nickinovich DG, et al. American Society of Anesthesiologists Task Force on Management of the Difficult Airway. Practice guidelines for management of the difficult airway: an updated report by the American Society of Anesthesiologists Task Force on Management of the Difficult Airway. Anesthesiology 2013 Feb;118(2):251-70.

5. Nørskov AK, Rosenstock CV, Wetterslev J, Astrup G, Afshari A, Lundstrøm LH. Diagnostic accuracy of anaesthesiologists' prediction of difficult airway management in daily clinical practice: a cohort study of 188064 patients registered in the Danish Anaesthesia Database. Anaesthesia 2015 Mar;70(3):272-81.

6. Burgers JS, Grol RP, Zaat JO, Spies TH, van der Bij AK, Mokkink HG. Characteristics of effective clinical guidelines for general practice. Br J Gen Pract 2003 Jan;53(486):15-9.

7. Leentjens AF, Burgers JS. [What factors are important for the successful implementation of guidelines?]. Tijdschr Psychiatr 2008;50(6):329-35.

8. WHO/IER/PSP/2008.09 (C) World Health Organization 2008. Patient Safety Workshop: LEARNING FROM ERROR.

9. Chrimes N, Fritz P. The Vortex Approach: Management of the Unanticipated Difficult Airway: (E-Book) Melbourne, Australia:
Monash Anaesthesia, 2013. At: http://www.vortexapproach.org/ Vortex_Approach/Vortex.html

10. Flin R, Fioratou E, Frerk C, Trotter C, Cook TM. Human factors in the development of complications of airway management: preliminary evaluation of an interview tool. Anaesthesia 2013 Aug;68(8):817-25.

11. Cook T, Woodall NM, Frerk C. 4th National Audit Project of the Royal College of Anaesthetists and the Difficult Airway Society. Major complications of airway management in the United Kingdom.

12. Brindley PG, Beed M, Duggan $\mathrm{LV}$, et al. Updating our approach to the difficult and failed airway: time to "stop and think". Can J Anaesth 2016 (publicado online Feb 2016).

13. Taha SK, El-Khatib MF, Baraka AS, Haidar YA, Abdallah FW, Zbeidy RA, et al. Effect of suxamethonium $v$ s rocuronium on onset of oxygen desaturation during apnoea following rapid sequence induction. Anaesthesia 2010 Apr;65(4):358-61.

14. Tang L, Li S, Huang S, Ma H, Wang Z. Desaturation following rapid sequence induction using 
succinylcholine vs rocuronium in overweight patients. Acta Anaesthesiol Scand 2011 Feb;55(2):203-8.

15. Kyle BC, Gaylard D, Riley RH. A persistent 'can't intubate, can't oxygenate' crisis despite rocuronium reversal with sugammadex. Anaesth Intensive Care 2012 Mar;40(2):344-6.

16. Neilipovitz DT, Crosby ET. No evidence for decreased incidence of aspiration after rapid sequence induction. Can J Anaesth 2007 Sep;54(9):748-64.

17. Piepho T, Cavus E, Noppens R, Byhahn C, Dörges V, Zwissler B, et al. Guideline of the German Society of Anesthesiology and Intensive Care Medicine. S1 Guidelines on airway management. Anaesthesist 2015 Dec;64(S1 Suppl 1):27-40.

18. Jensen AG, Callesen T, Hagemo JS, Hreinsson K, Lund V, Nordmark J; Clinical Practice Committee of the Scandinavian Society of Anaesthesiology and Intensive Care Medicine. Scandinavian clinical practice guidelines on general anaesthesia for emergency situations. Acta Anaesthesiol Scand 2010 Sep;54(8):922-50.

19. Field JM, Hazinski MF, Sayre MR, Chameides L, Schexnayder SM, Hemphill R et al. Part 1: executive summary: 2010 American Heart Association Guidelines for cardiopulmonary resuscitation and emergency cardiovascular care. Circulation 2010 Nov;122(18 Suppl 3):S640-56.

20. Mayglothling J, Duane TM, Gibbs M, McCunn M, Legome E, Eastman AL, et al. Eastern Association for the Surgery of Trauma. Emergency tracheal intubation immediately following traumatic injury: an Eastern Association for the Surgery of
Trauma practice management guideline. J Trauma Acute Care Surg 2012 Nov;73(5 Suppl 4):S333-40.

21. Recomendaciones de la Sociedad de Anestesiología de Chile para el manejo de la vía aérea difícil. Rev Chil Anest 2012;41:166-78.

22. Mosier JM, Joshi R, Hypes C, Pacheco G, Valenzuela T, Sakles JC. The physiologically difficult airway. West J Emerg Med 2015 Dec;16(7):1109-17.

23. Mort TC. Complications of emergency tracheal intubation: hemodynamic alterations-part I. J Intensive Care Med 2007 MayJun;22(3):157-65.

24. Weingart SD. Preoxygenation, reoxygenation, and delayed sequence intubation in the emergency department. J Emerg Med 2011 Jun;40(6):661-7.

25. Weingart SD, Trueger NS, Wong N, Scofi J, Singh N, Rudolph SS. Delayed sequence intubation: a prospective observational study. Ann Emerg Med 2015 Apr;65(4):349-55.

26. Leeuwenburg T. Airway management of the critically ill patient: modifications of the traditional rapid sequence induction and intubation. Critical Care Horizons 2015;1:1-10.

27. Mort TC. Emergency tracheal intubation: complications associated with repeated laryngoscopic attempts. Anesth Analg 2004 Aug;99(2):607-13.

28. Calder I, Yentis SM. Could 'safe practice' be compromising safe practice? Should anaesthetists have to demonstrate that face mask ventilation is possible before giving a neuromuscular blocker? Anaesthesia 2008 Feb;63(2):113-5.

29. Kheterpal S, Han R, Tremper KK, Shanks A, Tait AR, O'Reilly $\mathrm{M}$, et al. Incidence and predic- tors of difficult and impossible mask ventilation. Anesthesiology 2006 Nov;105(5):885-91.

30. Weingart SD, Levitan RM. Preoxygenation and prevention of desaturation during emergency airway management. Ann Emerg Med 2012 Mar;59(3):165-75.e1.

31. Patel A, Nouraei SA. Transnasal Humidified Rapid-Insufflation Ventilatory Exchange (THRIVE): a physiological method of increasing apnoea time in patients with difficult airways. Anaesthesia 2015 Mar;70(3):323-9.

32. Griesdale DE, Liu D, McKinney J, Choi PT. Glidescope ${ }^{\circledR}$ videolaryngoscopy versus direct laryngoscopy for endotracheal intubation: a systematic review and meta-analysis. Can J Anaesth 2012 Jan;59(1):41-52.

33. Bailly A, Lascarrou JB, Le Thuaut A, Boisrame-Helms J, Kamel T, Mercier E, et al. Clinical Research in Intensive Care and Sepsis Group. McGRATH MAC videolaryngoscope versus Macintosh laryngoscope for orotracheal intubation in intensive care patients: the randomised multicentre MACMAN trial study protocol. BMJ Open 2015 Dec;5(12):e009855.

34. Levitan RM, Mickler T, Hollander JE. Bimanual laryngoscopy: a videographic study of external laryngeal manipulation by novice intubators. Ann Emerg Med 2002 Jul;40(1):30-7.

35. Knill RL. Difficult laryngoscopy made easy with a "BURP". Can J Anaesth 1993 Mar;40(3):27982.

36. Gaitini L, Madrid V, Capdevila M, Ariño JJ. El tubo laríngeo.

Rev Esp Anestesiol Reanim 2008 Apr;55(4):232-41.

37. Ott T, Fischer M, Limbach 
T, Schmidtmann I, Piepho T, Noppens RR. The novel intubating laryngeal tube (iLTS-D) is comparable to the intubating laryngeal mask (Fastrach) - a prospective randomised manikin study. Scand J Trauma Resusc Emerg Med 2015 Jun;23(1):4451.

38. Biedler A, Wrobel M, Schneider S, Soltész S, Ziegeler S, Grundmann U. Randomized evaluation of the size 2 laryngeal tube and classical laryngeal mask airway in different head and neck positions in children under positive pressure ventilation. J Anesth 2013 Oct;27(5):657-62.

39. Schalk R, Scheller B, Peter N, Rosskopf W, Byhahn C, Zacharowski $\mathrm{K}$ et al. Larynxtubus II. Anaesthetist 2011;60(6):525-33.

40. Valero R, Mayoral V, Massó E, López A, Sabaté S, Villalonga $\mathrm{R}$, et al. Evaluación y manejo de la vía aérea dificil prevista y no prevista: adopción de guías de práctica. Rev Esp Anestesiol
Reanim 2008 Nov;55(9):56370.

41. Hagberg CA, Gabel JC, Connis RT. Difficult Airway Society 2015 guidelines for the management of unanticipated difficult intubation in adults: not just another algorithm. Br J Anaesth 2015 Dec;115(6):812-4.

42. Heard AM, Green RJ, Eakins P. The formulation and introduction of a 'can't intubate, can't ventilate' algorithm into clinical practice. Anaesthesia 2009 Jun;64(6):601-8.

43. Kristensen MS, Teoh WH, Baker PA. Percutaneous emergency airway access; prevention, preparation, technique and training. Br J Anaesth 2015 Mar;114(3):357-61.

44. Heard A. Percutaneous emergency oxygenation strategies in the can't intubate - can't oxygenate scenario. Smashworks Editions 2013. Disponible en https:// www.smashwords.com/books/ view/377530
45. Cook TM, Andrade J, Bogod DG, Hitchman JM, Jonker WR, Lucas N, et al. Royal College of Anaesthetists and the Association of Anaesthetists of Great Britain and Ireland. The 5th National Audit Project (NAP5) on accidental awareness during general anaesthesia: patient experiences, human factors, sedation, consent and medicolegal issues. Anaesthesia 2014 Oct;69(10):1102-16.

46. Pandit JJ, Popat MT, Cook TM, Wilkes AR, Groom P, Cooke $\mathrm{H}$, et al. The Difficult Airway Society 'ADEPT' guidance on selecting airway devices: the basis of a strategy for equipment evaluation. Anaesthesia 2011 Aug;66(8):726-37.

47. Hagberg CA, Gabel JC, Connis RT. Difficult Airway Society 2015 guidelines for the management of unanticipated difficult intubation in adults: not just another algorithm. Br J Anaesth 2015 Dec;115(6):812-4. 